\title{
Determinación del espesor óptico de aerosol en la Estación Antártica Peruana Machu Picchu
}

\section{Determination of aerosol optical depth in the Machu Picchu Peruvian Antarctic Station}

\author{
Julio Ángeles Suazo \\ Universidad Alas Peruanas
}

\section{RESUMEN}

Objetivos: Determinar el espesor óptico de los aerosoles (EOA) durante los meses de enero 2007 y 2008 y febrero de 2013 en la Estación Científica Antártica Machu Picchu (ECAMP). Métodos: La investigación fue descriptiva comparativa. El principal instrumento para evaluar el EOA fue el fotómetro solar SP02-L para mediciones directas del sol con 4 sensores centrados en las longitudes de onda $412,500,675$ y $862 \mathrm{~nm}$. Solo fueron utilizados registros con un ángulo cenital solar inferior a $80^{\circ}$ y con cero de cobertura nubosa $30^{\circ}$ alrededor del Sol. Estas mediciones fueron comparadas, a través de la prueba de Pearson, con EOA a $550 \mathrm{~nm}$ de los sensores satelitales MODIS (Moderate Resolution Imaging Spectroradiometer). Resultados: Se determinó una máxima y mínima media de EOA de 0,1061 y 0,0616, respectivamente. Se reportó un coeficiente de correlación con el sensor MODIS, tanto del satélite Aqua y Terra, siendo 0,0284 y $0,0059(p<0,05)$, respectivamente. La misma correlación fue evaluada con el Índice de Aerosol del sensor OMI (Ozone Monitoring Instrument) resultando una mayor correlación, $0,3606(p<0,05)$. Comparando el EOA resultante en la ECAMP con las demás estaciones antárticas se encontraron valores similares alrededor de 0,06 , especialmente cercanas a zonas costeras como Neumayer y Aboa. Conclusiones: Los datos obtenidos en la ECAMP en los años 2007, 2008 y 2013 no evidencian una variación significativa respecto al espesor óptico de aerosoles. Los altos valores obtenidos en la ECAMP comparados con

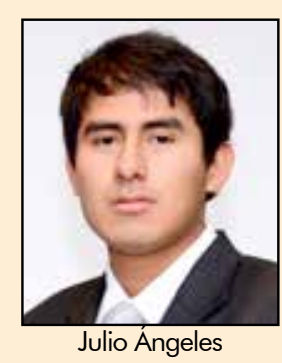

julio_as_1@hotmail.com

las estaciones de Aboa y Neumayer pueden tener su explicación en las mediciones manuales realizadas en comparación con las automáticas de las otras estaciones.

Palabras clave: Espesor óptico de aerosol, Estación Científica Antártica Machu Picchu, fotómetro solar. 


\section{ABSTRACT}

Objectives: To determine the aerosol optical depth (AOD) during january 2007 and 2008 and february 2013, at Machu Picchu Peruvian Scientific Antarctic Station (ECAMP). Methods: The research was descriptive and comparative. The main instrument to evaluate AOD was the SP02-L solar photometer for solar direct measurements with 4 sensors centered at the wavelengths of $412,500,675$ y $862 \mathrm{~nm}$. Only were used records with solar zenith angle lower than $80^{\circ}$ and with cloud coverage of zero $30^{\circ}$ around the sun. These measurements were compared through the Pearson test with AOD at $550 \mathrm{~nm}$ from MODIS (Moderate Resolution Imaging Spectroradiometer) satellites sensors. Results: It was determined a maximum and minimum mean of $A O D$ at 0,1061 and 0,0616 respectively. It was reported a correlation coefficient with the MODIS sensor, in both the Aqua and Terra satellites, being 0,0284 and 0,0059 $(p<0,05)$ respectively. The same correlation was evaluated with the Aerosol Index from OMI (Ozone Monitoring Instrument) sensor resulting a higher correlation, 0,3606 $(p<0,05)$. Comparing the resulting AOD at the ECAMP with other Antarctic stations, it was found similar values around 0,06, especially with the ones close to coastal areas like Neumayer and Aboa. Conclusions: The obtained data at ECAMP during the years 2007, 2008 and 2013 don't evidence a significant variation regarding to the aerosol optical depth. The high values obtained at ECAMP compared with the Aboa and Neumayer stations can have its explanation in the manual measurements realized in comparison with the automatic ones from other stations.

Keywords: Aerosol optical depth, Machu Picchu Peruvian Scientific Antarctic Station, solar photometer.

\section{INTRODUCCIÓN}

Los aerosoles atmosféricos o material particulado, son un conjunto de partículas gaseosas, sólidas o líquidas suspendidas en la atmósfera (1) y juegan un rol importante en el cambio climático (2), porque influyen en el clima de manera directa mediante la dispersión y absorción de la radiación solar produciendo el enfriamiento y calentamiento, respectivamente (3); y de manera indirecta, el tamaño de los aerosoles actúan en la formación de distintos tipos de nubes, ya que facilitan el proceso de condensación de vapor de agua en la atmósfera actuando como núcleos de condensación para la formación de nubes - modificando las propiedades ópticas y período de vida de las nubes (4).

Es tanto su importancia que la Organización Meteorológica Mundial (OMM) ha incluido el espesor óptico de aerosoles en el listado básico de medidas del Programa de Vigilancia Atmosférica Mundial, ya que alteran en forma directa el clima debido a la dispersión y absorción de la radiación y la influencia en el albedo planetario en el sistema climático. Los efectos indirectos incluyen la influencia de los aerosoles antropogénicos en núcleos de condensación para formación de nubes (5).

Los aerosoles también pueden producir un gran efecto en la salud, existe abundante evidencia que muestra una fuerte correlación estadística positiva entre la concentración de PM10 y la mortalidad diaria (6), y entre PM10 y las enfermedades respiratorias agudas bajas en niños menores de 14 años (7).

Aún no se conoce la cantidad de aerosoles contribuidos por los humanos en la región antártica peruana; sin embargo, existen estudios que demuestran que el transporte de contaminantes del aire pueden alcanzar sitios tan alejados como la Antártida (2), y que la composición química varía mucho de unas partículas a otras, dependiendo del origen y pudiendo ser trasladados a grandes distancias por acción de los vientos. Por 
esta razón, es de interés el estudio de los aerosoles para identificar una influencia del transporte de contaminantes e identificar las fuentes más importantes o algún efecto antropogénico negativo en la calidad de aire (8) y comparar con las mediciones de otras estaciones antárticas que están en la zona costera para lograr uniformizar la metodología en la determinación del EOA (9).

El problema que conduce al presente trabajo es: ¿Cuál es la variabilidad del espesor óptico de aerosol existente en los meses de enero de 2007 y 2008, así como febrero de 2013, en la Estación Antártica Peruana Machu Picchu?

El objetivo fue evaluar la variabilidad del espesor óptico de los aerosoles precisamente en tales meses, a raíz de la XVII, XVIII y XXI Expedición Antártica Peruana realizada durante las referidas fechas en la Estación Científica Antártica Machu Picchu (ECAMP). El trabajo busca con ello determinar las principales propiedades ópticas de los aerosoles y desarrollar una evaluación para comprobar si los aerosoles contribuyen a un enfriamiento o calentamiento en el sistema terrestre, sea para mejorar el entendimiento de los impactos de su variación como para implementar sus valores en modelos climatológicos, puesto que el estudio de los aerosoles aún tiene un bajo nivel de entendimiento (2).

\section{MATERIAL Y MÉTODOS}

Las mediciones se dieron en la Estación Científica Antártica Peruana Machu Picchu (ECAMP) ubicada en la Punta Crespín,

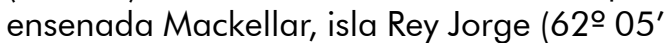
$30^{\prime \prime} \mathrm{S}$ y 58 28' 16" W a $6 \mathrm{msnm}$ ) durante los meses de enero y febrero para los años 2007(ANTAR XVII), 2008(ANTAR XVIII) y 2013 (ANTAR XXI).

Para la determinación del espesor óptico de aerosol (EOA), se utilizó un fotómetro solar SP02-L (Middleton Inc. Australia) (10); este instrumento es utilizado para observaciones de aerosoles, ozono y vapor de agua. Para la configuración del aerosol, el SP02-L tiene 4 canales en las longitudes de onda de $412,500,675$ y $862 \mathrm{~nm}$, correspondientes a la radiación visible. Estos espectropiroheliometros se encuentran actualmente alineados en un recinto herméticamente cerrado, los mismos que actúan de modo simultáneo cuando el instrumento es alineado directamente al Sol, acción que permite una resolución fina de indicación del espesor óptico de aerosol en la atmósfera.

La versión SP02-L tiene un ancho de banda de $10 \mathrm{~nm}$ y un campo de visión de 2,5을 las señales de salida tienen un rango de $-0,05$ a 4,50 VDC, adicionalmente tiene una salida de temperatura interna $\left(10 \mathrm{mV} / 1^{\circ} \mathrm{C}\right)$. La temperatura de operación del fotómetro solar es de $-30^{\circ} \mathrm{C}$ a $70^{\circ} \mathrm{C}$.

También se recurrió a los satélites Aqua y Terra que cuentan con el sensor MODIS (Moderate Resolution Imaging Spectroradiometer) que nos brindan el EOA a $550 \mathrm{~nm}$ perteneciente al área de 61680 a 62680 latitud Sur y al de 58,40 a 59,40 longitud Oeste.

Las concentraciones de la columna total de ozono para la determinación del espesor óptico de aerosol se obtuvieron del sensor OMI (Ozone Monitoring Instrument) de la NASA que brinda la variable denominada índice de aerosol (IA) desde el año 2004.

\section{Espesor óptico de aerosol (EOA)}

El EOA como se indica en la ecuación 1 se estima a partir del espesor óptico total $(\tau)$, el cual es la suma de los espesores ópticos del aire (referido como dispersión Rayleigh, $\left(\tau_{\text {Rayleigh }}\right)$, ozono $\left(\tau_{\text {ozono }}\right)$ y aerosoles (EOA) $(11)$. Este cálculo se determina para cada una de las longitudes de onda correspondientes al fotómetro solar SP02-L, siendo 412, 500 675 y $862 \mathrm{~nm}$.

$\mathrm{EOA}=\tau-\tau_{\text {Rayleigh }}-\tau_{\text {ozono }}$

La secuencia de ecuaciones se basa en 
el cálculo de los tres primeros espesores ópticos. La determinación del espesor óptico total está basada en la Ley de Lambert-Beer (ecuación 2), que explica la atenuación de la radiación solar al paso por la atmósfera (9).

$$
\tau=\frac{\ln \left(R I_{0 \lambda} / I_{\lambda}\right)}{m}
$$

Donde $\mathrm{R}$ es el factor de corrección distancia sol-tierra; $I_{\lambda}$ es la intensidad de radiación observada a una longitud de onda $(\lambda)$, a nivel de la superficie del suelo; $I_{0 \lambda}$ es la intensidad de radiación en el tope de la atmósfera, obtenido gráficamente por el método de Langley (12), para una $\lambda$ en particular; y $\mathrm{m}$ es la masa del aire determinado por la secante del ángulo cenit solar.

El método de Langley está basado en el principio de extinción espectral y la ley de Beer- Lambert- Bouguer. En este método las observaciones de radiación son ploteados como una función de la masa de aire. La pendiente de la regresión lineal resultante es $\left(\tau_{\text {total }}\right)$ y la radiación correspondiente a $\mathrm{m}$ igual a cero es la $\mathrm{I}_{0 \lambda}$.

El espesor óptico del aire, conocido como el de Rayleigh, es calculado utilizando la ecuación 3 (13):

$\tau_{\mathrm{R}}(\lambda)=(a+b H) \lambda^{-(c+d \lambda+e / \lambda)} P / P_{S}$

Donde, $a=0,00864, \quad b=6,5^{*} 10-6$, $c=3,916, d=0,074, e=0,05 ; \mathrm{H}$ es la altura del fotómetro solar SP02-L en kilómetros; $P$ es la presión de sitio ( $~ 986$ en mb); Ps, presión atmosférica estándar 1013,25 mb; y $\lambda$ la longitud de onda en micrómetros (13).

El espesor óptico de ozono está en función de la concentración de la columna total de ozono $(\Omega)$ en Unidades Dobson obtenido con el sensor OMl y el coeficiente de absorción de ozono $(\sigma)$ según la ecuación 4 .

$$
\tau_{03}=\frac{\Omega \times \sigma}{1000}
$$

Para las longitudes de onda de 412, 500, 675 y 862, los coeficientes de absorción son 0,000295974375, 0,031997375, 0,0401001875 y 0,00247309125 , respectivamente (14).

\section{RESULTADOS}

\section{Mediciones de intensidad incidente}

Para el procesamiento de datos en el cálculo del espesor óptico se realizaron lecturas cada 10 segundos, con cero cobertura nubosa alrededor del Sol, en un promedio de 82 lecturas por cada longitud de onda durante los meses de enero de 2007, enero de 2008 y enero-febrero de 2013.

Estudios realizados indican que al menos 20 ajustes son necesarios, porque variaciones monótonas en el EOA dan lugar a errores sistemáticos en la determinación de la intensidad inicial $I_{0 \lambda}$ por el método de Langley (15), debido a que estos errores son imposibles de detectar en un solo ajuste (16). Por ello, para asegurar una adecuada $I_{0 \lambda}$ se realizaron 7 ajustes a partir de los cuales se obtuvo un promedio, ajuste recomendado por otros investigadores (17) con la finalidad de determinar el EOA con un error absoluto de 0,01 - 0,02. En condiciones de baja turbidez, este error absoluto puede significar un error relativo superior al $10 \%$ en el EOA (18).

En la obtención de la curva de Langley consiste en obtener medidas de voltajes con una variación continua de la columna de masa de aire entre el fotómetro solar y el sol. Y para la obtención de la intensidad en la parte superior de la atmosfera se hizo una extrapolación de la señal espectral $I_{\lambda}$ para la obtención de la constante $I_{0 \lambda}$ cuando la masa del aire $(\mathrm{m})$ es cero $(\mathrm{m}=$ $0)$, de acuerdo con la ecuación 5, para las longitudes de onda de 412, 500, 675 y 862 $\mathrm{nm}$ como se muestra en la figura $\mathrm{N}^{\circ} 1$. 

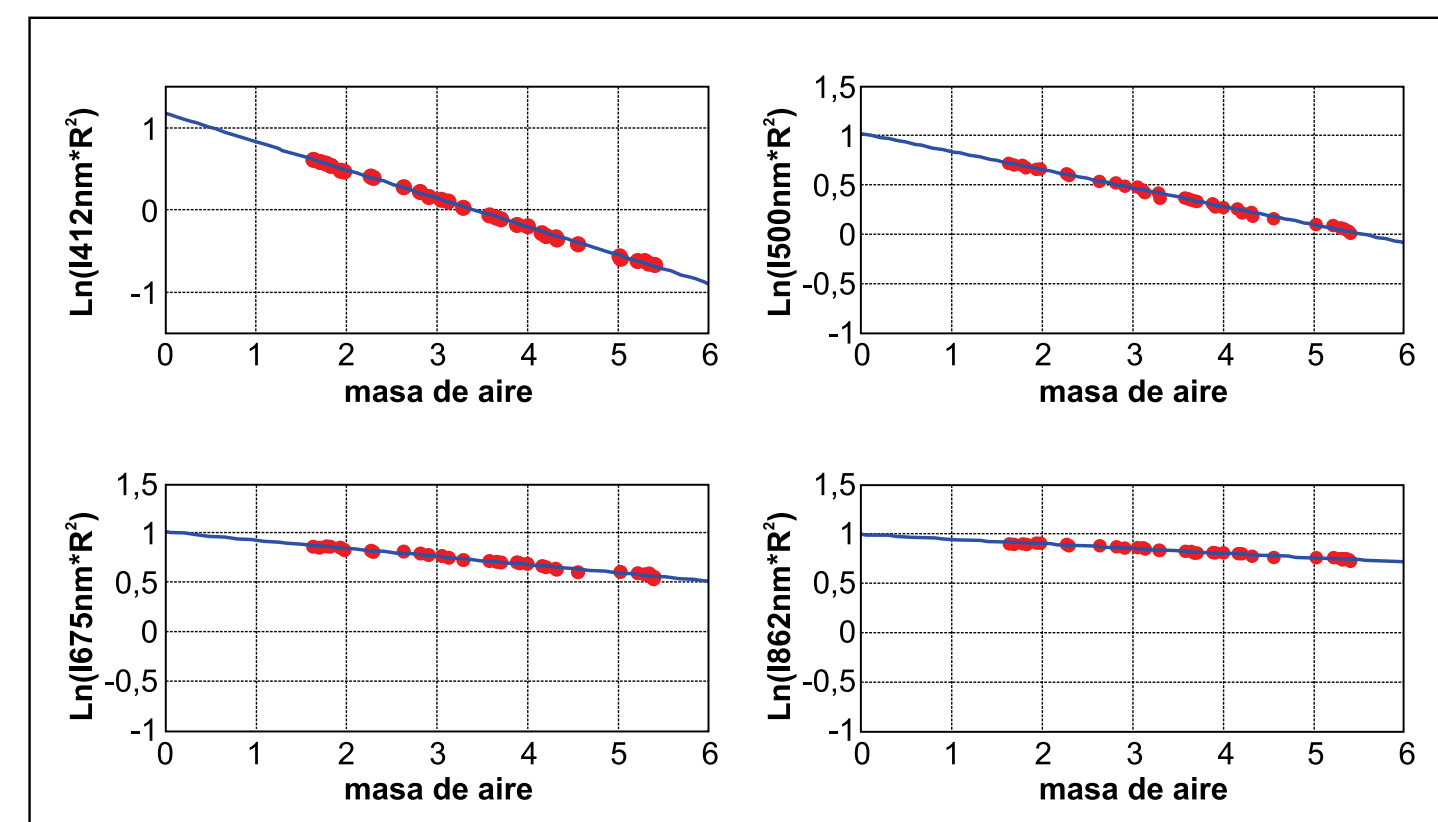

Figura $\mathrm{N}^{\circ}$ 1: Gráfico esquemático de la curva de Langley-plot para la obtención de la intensidad en la parte superior de la atmósfera

Tabla $N^{\circ} 1$ : Espesor óptico de Rayleigh y ozono

\begin{tabular}{lllll}
\hline Longitud de onda & $412 \mathrm{~nm}$ & $500 \mathrm{~nm}$ & $675 \mathrm{~nm}$ & $412 \mathrm{~nm}$ \\
\hline Espesor óptico de ozono & 0,000078911 & 0,0085 & 0,0107 & 0,000078911 \\
Espesor óptico de Rayleigh & 0,2367 & 0,1154 & 0,0373 & 0,2367 \\
\hline
\end{tabular}

$\ln \left(I(\lambda) R^{2}\right)=\ln \left(I_{0}(\lambda)-m \tau(\lambda)(5)\right.$

Así, se obtiene las intensidades en la parte superior de la atmósfera que representan la radiación incidente para una atmósfera limpia sin contaminantes obteniendo las intensidades iniciales de 3,2143, $2,7661,2,7570$ y 2,6836 voltios para las longitudes de onda 412, 500, 675 y 862 $\mathrm{nm}$, respectivamente, que nos indica la radiancia que incide cuando la masa del aire es cero.

\section{Espesores ópticos de ozono y Rayleigh}

En relación con los espesores ópticos, la tabla $\mathrm{N}^{\circ} 1$ muestra un resumen de los valores encontrados para cada tipo de espesor óptico, del total de las observaciones realizadas con ángulos cenit solar inferior a $80^{\circ}$. Estos valores son normalmente pequeños en comparación con otras ubicaciones no polares, las condiciones de clima y ubicación de la región antártica condicionan a que estas propiedades ópticas sean menores (19).

\section{Espesor óptico de aerosol determinado con el SP02-L}

La figura $\mathrm{N}^{\circ} 2$ corresponde a las mediciones promedio de los años 2007, 2008 y 2013, con cero de cobertura solar durante las horas de la mañana; en la cual, muestra un resumen de los valores encontrados para cada longitud de onda del espesor óptico del total de las observaciones realizadas con una masa de aire óptica de 2 a 5 . Se puede observar que el espesor óptico de aerosol disminuye a medida que aumenta la longitud de onda, mientras que la interacción entre la radiación y los aerosoles se incrementa, la longitud de 


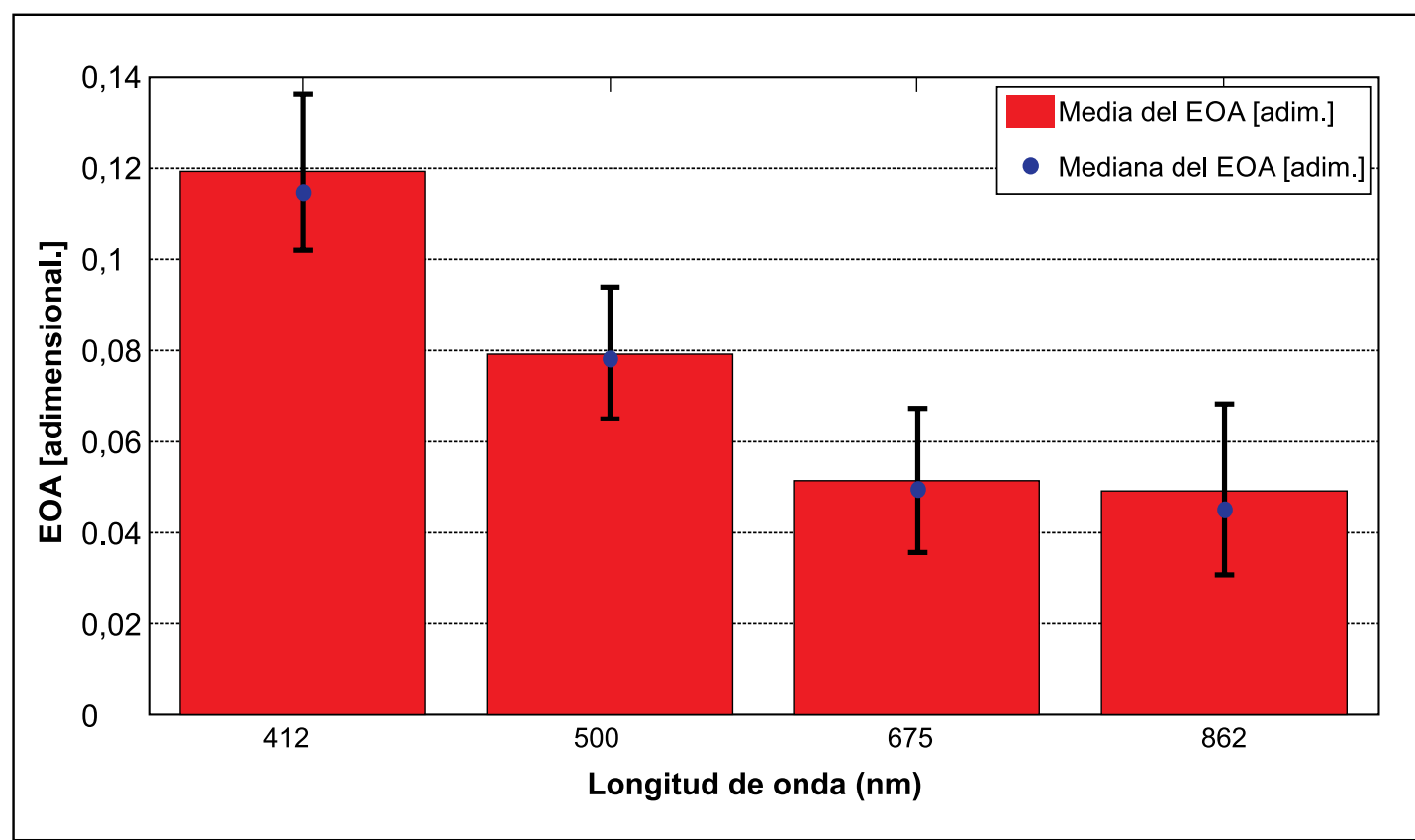

Figura $\mathrm{N}^{\circ}$ 2: Variación del espesor óptico para cada longitud de onda

onda de $862 \mathrm{~nm}$ tiene mayor variación por efecto de los aerosoles, el valor promedio y coeficiente de variación de EOA a 500 $\mathrm{nm}$ y 862 son de 0,0794 $\pm 0,0144$ y $0,0493 \pm 0,0187$ respectivamente. Estos resultados de espesor óptico de aerosol (EOA) están dentro del rango de los hallados en regiones polares por otros grupos (19), siendo menores en relación con los encontrados en ciudades urbanas entre 0,25 a 1,7 (20) y mucho menores que los registrados en lugares de quema de la biomasa, obteniéndose valores en el rango de 0,6 a 2,4 para similares longitudes de onda (21).

En la figura $\mathrm{N}^{\circ} 3$ se muestra una serie histórica de los valores del espesor óptico

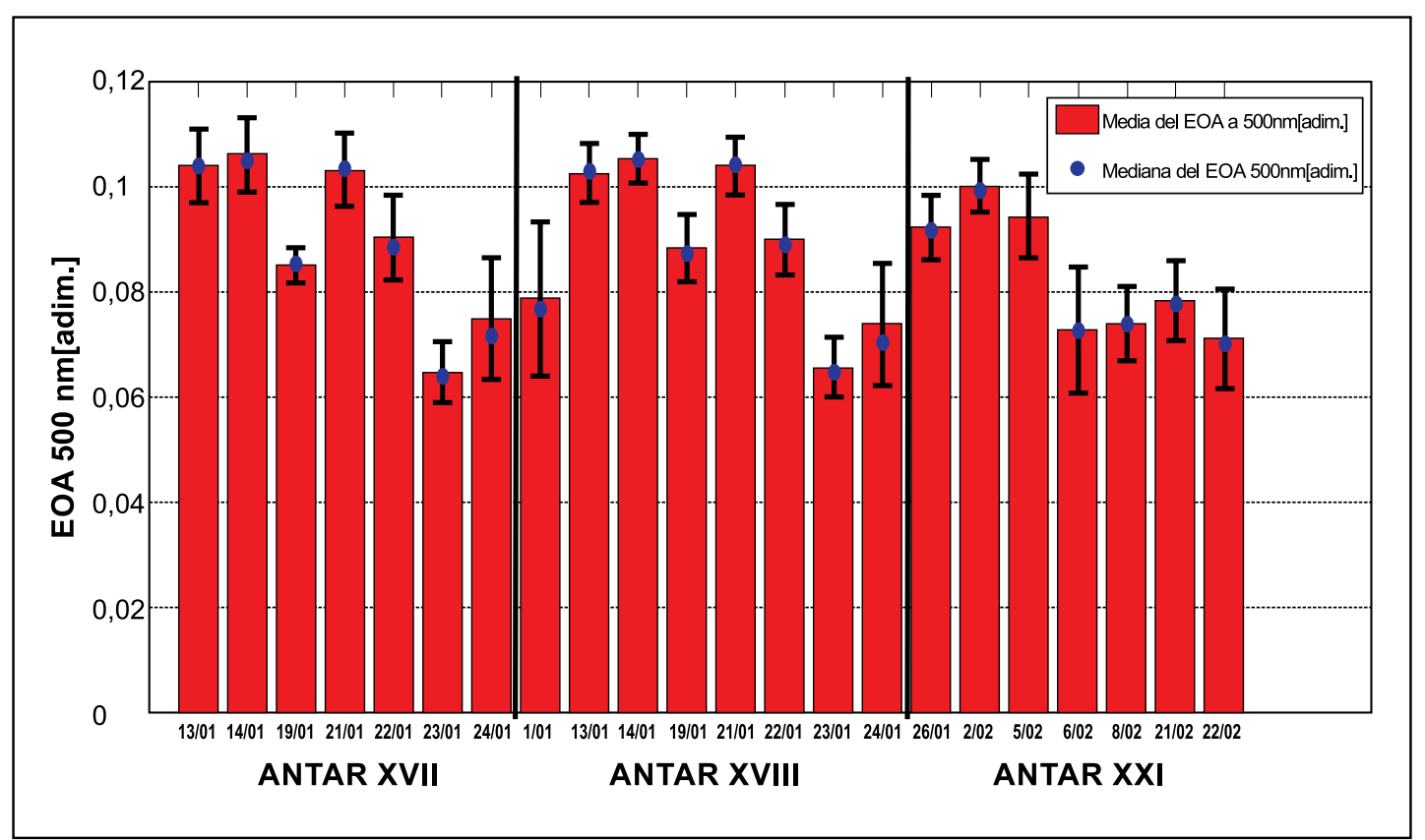

Figura $N^{\circ}$ 3: Variación diaria del EOA a 500 nm para los años de 2007, 2008 y 2013 


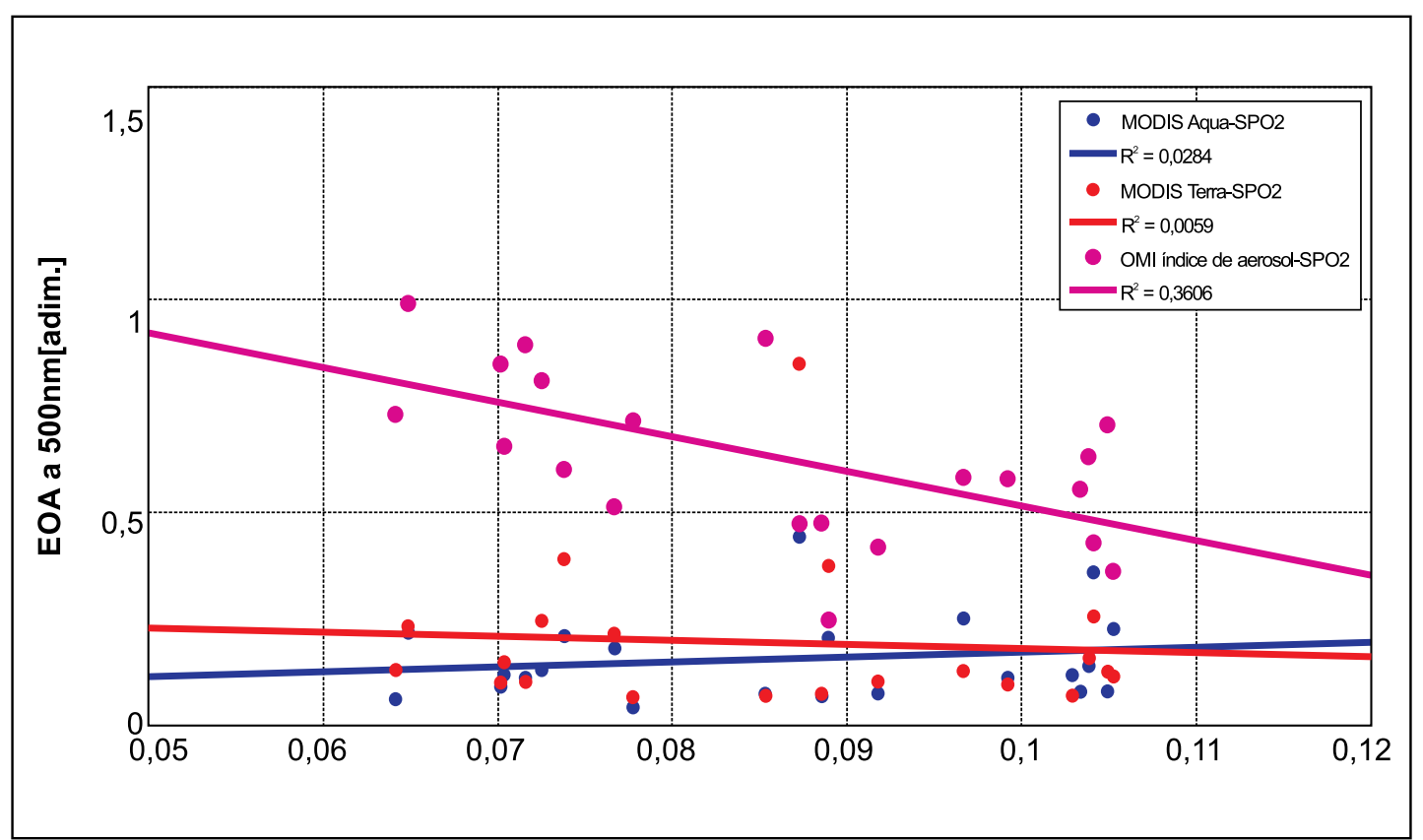

Figura $\mathrm{N}^{\circ}$ 4: Comparación entre datos obtenidos con los sensores MODIS y OMI con el fotómetro solar SPO2-L

de aerosoles durante los años 2007, 2008 y 2013 . Como se puede observar la mediana del espesor óptico de aerosoles, varía entre 0,0641 a 0,1052; siendo típico por las condiciones de turbidez atmosférica del sitio en este tiempo del año (22).

La campaña fue planificada con el objetivo principal de cuantificar la comparacion que existe entre los distintos años de mediciones donde se dio una máxima y mínima media del espesor óptico de aerosoles a $500 \mathrm{~nm}$ de 0,1061 y 0,0646 para el 14 y 23 de enero del año 2007 respectivamente.

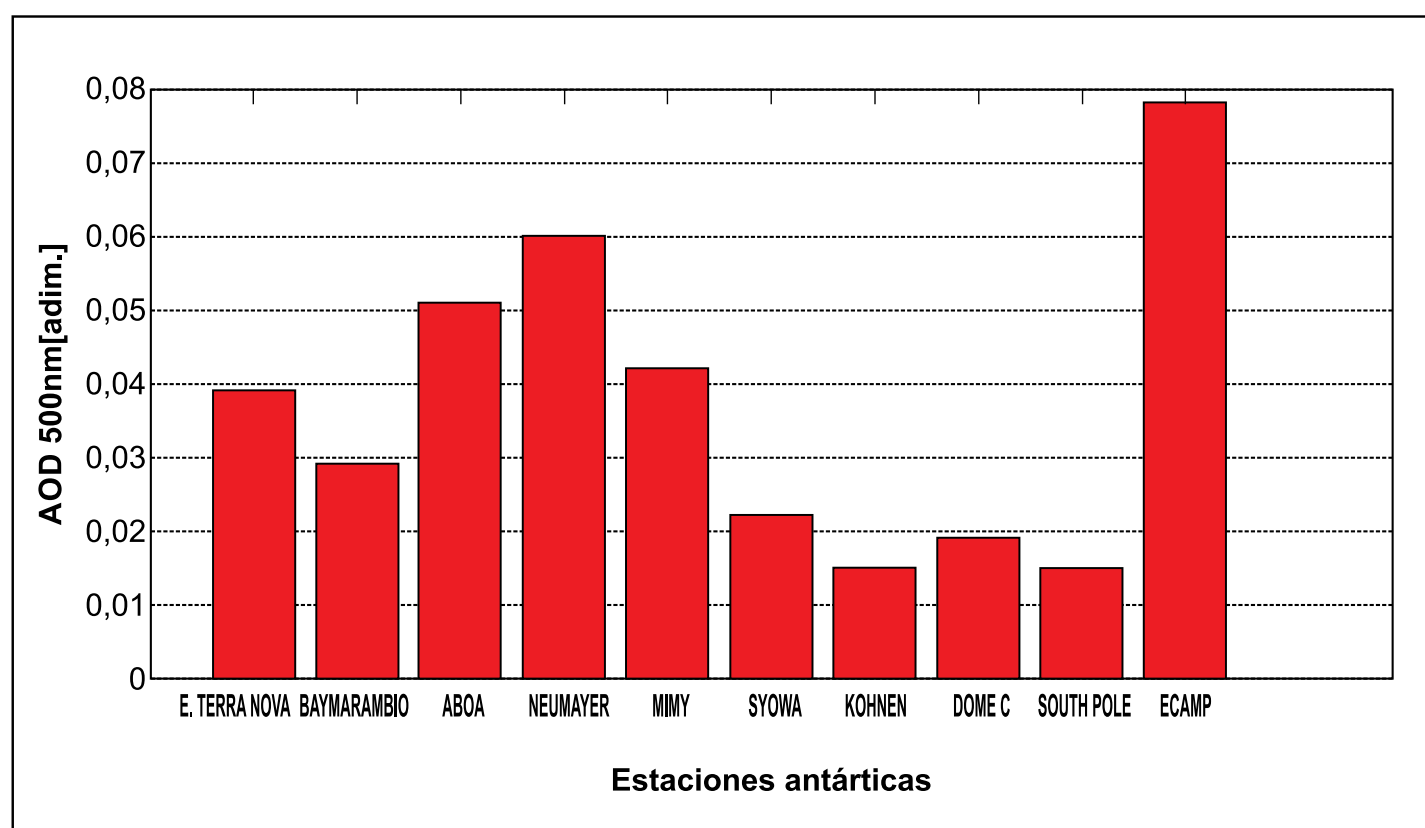

Figura $\mathrm{N}^{\circ}$ 5: Comparación entre datos obtenidos con los sensores MODIS y OMI con el fotómetro solar SP02-L 


\section{Comparación de espesores ópticos de aerosol obtenidos con el sensor MODIS y SPO2}

La figura $\mathrm{N}^{\circ} 4$ corresponde a las mediciones de los años 2007, 2008 y 2013, para el sensor MODIS a $550 \mathrm{~nm}$ que pertenece a los satélites Aqua y Terra, y para el fotómetro solar SP02-L a $500 \mathrm{~nm}$, cuyo proceso se comparó ambas mediciones para los 3 años y quedó determinado que el grado de correlación entre el sensor MODIS (perteneciente a los satélites Aqua y Terra) y el fotómetro solar SP02 es bajo, de 0,0284 y 0,0059, respectivamente, por el que se recomienda una estudio detallado del sensor MODIS. En cambio, en la comparación del espesor óptico de aerosol (EOA) que se determinó con el fotómetro solar SP02 a 500 nm, y el Índice de Aerosol (IA) que se obtuvo mediante el sensor OMl a $360 \mathrm{~nm}$ se determinó un coeficiente de correlación de 0,3606 con un $p$-value $<0,05$.

\section{DISCUSIÓN}

En la figura $N^{\circ} 5$, considerando los valores obtenidos, la Estación Científica Antártica Machu Picchu presenta una alta mediana de EOA de 0,0781 como las estaciones de Neumayer y Aboa de 0,06 y 0,0551, respectivamente a $500 \mathrm{~nm}$, ya que pertenecen a la zona costera donde se da mayor influencia de aerosoles marinos. En cambio, los que no pertenecen a la zona costera presentan valores mínimos como la estación de Kohnen y South Pole de 0,015 cada una (22).

La comparación entre la ECAMP con las otras estaciones ofrece la evidencia de las diferencias considerables de las propiedades ópticas de los aerosoles. Es cierto que el aire es limpio en regiones polares, pero tienen una mezcla de partículas muy pequeñas principalmente de origen marino y a las condiciones de turbidez que suelen aplicar en verano y en otoño los fuertes cambios debidos al transporte de bruma y polvo (23).

El impacto radiativo directo de los aerosoles polares en la superficie y en la parte superior de la atmosfera necesitan ser estudiados más detenidamente a través de estudios teóricos sobre las propiedades radiativas de los aerosoles. La verificación de los resultados teóricos requiere el monitoreo continuo de las propiedades ópticas de los aerosoles, como determinar los modelos más realistas que sean de reflectancia anisotropía de la superficie para representar las diferentes albedos de superficie y características del mar y tierra en las regiones polares, como lo definió Ricchiazzi (24), también es necesario el factor de calibración para la determinación del EOA como las correcciones del espesor óptico de Rayleigh (25).

El trabajo permitió cuantificar el EOA en la ECAMP, con la finalidad de proveer y contribuir al estudio del forzamiento radiativo y a las propiedades ópticas de los aerosoles en zonas polares, e implementar estaciones de monitoreo continuo del EOA para realizar comparaciones con los datos satelitales, y obtener una nueva metodología para la obtención del EOA por sensoramiento remoto por satélite, debido a que las incertezas en los espesores ópticos obtenidos por sensores en satélites depende fuertemente de la calidad de la información sobre las propiedades ópticas de las partículas de los aerosoles, ya que en la presente investigación dio valores muy elevados de espesores ópticos obtenidos con el sensor MODIS, en relación con los valores medidos con el fotómetro solar en superficie.

Algunas conclusiones son:

Los datos obtenidos en la ECAMP en los años 2007, 2008 y 2013 no evidencia una variación significativa respecto al espesor óptico de aerosoles.

Los altos valores obtenidos en la ECAMP comparados con las estaciones de Aboa y Neumayer se debe a que en la ECAMP 
los valores obtenidos fueron medidos manualmente en comparación con las mediciones automáticas en las otras estaciones. También estos valores podrían haber sido influenciadas por sales marinas ya que la ECAMP pertenece a una zona costera.

Los valores de EOA contribuirá a la determinación del balance radiativo en el sistema climático antártico, también contribuirá a determinar las incertezas de los espesores ópticos obtenidos por sensores en satélites dependientes fuertemente de la calidad de la información sobre las propiedades ópticas de las partículas de los aerosoles.

\section{Agradecimientos}

Agradecimiento al Instituto Antártico Peruano por su apoyo en la realización de las mediciones y elaboración del presente artículo como parte de la expedición antártica. Asimismo a los ingenieros Luis Suárez Salas y Ana Contreras Marín por su apoyo técnico.

\section{REFERENCIAS BIBLIOGRÁFICAS}

1. Vergaz B. Propiedades ópticas de los aerosoles atmosféricos. Caracterización del área del Golfo de Cádiz, Valladolid. [Tesis Doctoral]: Universidad de Valladolid; 2001.

2. Foster P, Ramaswamy. IPCC, Changes in atmospheric constituents and in radiative forcing. UNIVERSITY PRESS CAMBRIDGE. NEW YORK, USA; 2007.

3. Andreae M. Climatic effects of changing atmospheric aerosol levelsen World Survey of Climatology. Elsevier.1995; 16: 341-392.

4. Raes F, Dingenen R, Vignati E, Wilson J, Putaud J, Sinfeld J, Adams M. Formation and cycling of aerosols in the global troposphere. Atmos. Environ. 2000; 34: 4215-4240.
5. WMO. Report of the WMO.WMO aerosol measurement procedure; 2003

6. Ostro B, Eskeland G, Sánchez J, Feyzioglu T. Air Pollution and Health Effects: A Study of Medical Visits among Children in Santiago, Chile. Environmental Health Perspectives. 1999; 107 (1):69-73.

7. Ostro B, Sánchez J, Aranda C, Eskeland G. Air Pollution and Mortality: Results from a Study of Santiago, Chile. Journal of Exposure Analysis and Environmental Epidemiology. 1996; 6(1): 97-114.

8. Kirchhoff V, Silva A, Costa C, PesLeme N, Pavao H, Zarratti F. UV-B optical thickness observations of the atmosphere. Journal of Geophysical Research. 2001; 106: 2963-2973.

9. Tomasi C, Vitale V. Calculation of the relative optical mass functions for air, water vapor, ozone and nitrogen dioxide in the Antarctic and Arctic Atmospheres. 7th Workshop Italian Research on Antarctic Atmosphere, Conference Proceedings. Italian Physical Society, Bologna, Italy. 1997; 62: 22-24.

10. Guía de usuario del fotómetro solar modelo SP02-L de Middleton Imc; 2004

11. Bodhaine B, Wood N, Dutton E, Slusser J. On rayleigh Optical Depth Calculations. Journal Atmos. and Ocean. 1999; 16: 1854-1864.

12. Reagan J, Scott-Fleming I, Herman BR. Recovery of spectral optical, depth and zero - air mass solar spectral irradiance under conditions of temporally varying optical depth proceedings of IGARSS'84 Symposium. Strasbourg. 1984; 215: 455-459.

13. Liou K. An introduction to atmospheric radiation. 2da. ed. New York: academic Press; 2007.

14. Michalsky J. Aerosol optical depth valueadded product. Climate Research. 2013; 129: 1-32.

15. Reagan J, Thomason L, Herman B, Palmer J. Assessment of atmospheric limitations on the determination of the solar spectral constant from ground-based spectroradiometer measurements. Geosci. Remote. 1986; 24: 258-265.

16. Terez E, Terez G. A method to determine 
atmospheric optical depth using observations of direct solar radiation. J. Geophys. 2003; 108 (D22): 1-6.

17. Slusser J, Gibson J, Bigelow D,Kolinski D, Disterhoft P, Lantz K, Baubien A. Langley method of calibrating UV filter radiometers. J. Geophys. 2000; 105 (D4): 4841-4849.

18. Wehrli Ch. Calibration of filter radiometers for determination of atmospheric optical depth. Metrologia. 2000; 37 (5): 419-422.

19. Stone R. Monitoring aerosol optical depth at Barrow, Alaska and South Pole; Historical overview, recent results, and future goals. Coop. Res. in Environ. Scien. 2002; 80: 123-144.

20. Castro T, Madronich S, Rivale S, Muhlia A, Mar B. influence of aerosols on photochemical smog in Mexico City. Atmospheric Environment. 2001; 35: 1765-1772.

21. Eck T, Holben B, Reid J, O'Neill N, Schafer J, Dubovik O, Simimov A, Yamasoe $M$ y Artaxo P. High aerosol optical depth biomass burning events: A comparison of optical properties for different source regions. Geophysical Research Letters. 2003; 30(20): 20352044.

22. Dutton E, Reddy P, Ryan S, Tomasi C. Aerosol in polar regions; A historical overview based on optical depth and in situ observations, J. Geophys. Res. 2007; 112: 1-28.

23. Shaw G. Atmospheric turbidity in the Polar Regions. J. Appl. Meteorol. 1982; 21: 1080 - 1088.

24. Ricchiazzi P, Gautier C. The effect of non-Lambertian surface reflectance on aerosol radiative forcing. U.S. Dep. of Energy Daytona Beach. 2005; 14- 18.

25. Mazzola M, Stone R. Evaluation of sun photometer capabilities for retrievals of aerosol optical depth at high latitudes: The POLAR-AOD intercomparison campaigns. Atmos. Environ. 2012; 52:4-17. 